\title{
The Impact of Malingering on Inpatient Assault Rates
}

\section{Julie S Costopoulos*, Darby Proctor, Alyssa M Johnson and Skyler G Roberts}

Florida Institute of Technology, Melbourne, Florida USA

*Corresponding Author: Julie S Costopoulos, Florida Institute of Technology, Melbourne, Florida USA.
Received: January 07, 2021

Published: May 20, 2021

(C) All rights are reserved by Julie $\mathbf{S}$

Costopoulos., et al.

\begin{abstract}
Assaults in forensic psychiatric hospitals are costly and damaging to the goals of treatment. This study examines the hypothesis that malingerers were responsible for a significantly higher rate of assaults. Individuals diagnosed as malingerers $(n=112)$ were matched with genuinely mentally ill individuals $(n=112)$ based on legal status, length of stay, closest age, and gender. Significantly more malingerers committed at least one assault, and committed a greater rate of assaults than patients with genuine clinical syndromes. Young age and personality disorders were also associated with higher frequency of assaults. Findings support the need to reform methods utilized to identify malingerers prior to commitment to an inpatient setting, to reduce the negative impact of assaults perpetrated by malingerers' unnecessary hospital stays.
\end{abstract}

Keywords: Inpatient Assaults; Malingerers; Forensic Hospitalization

Impact of aggression in inpatient settings

Violence in healthcare settings is a significant concern, posing a threat to both the mental and physical safety of healthcare providers, staff, and other patients. Violence in this setting leads to increased burnout in staff, posing a widespread threat to the quality and quantity of healthcare providers available to serve the public [1]. Staggs [2] found that $75 \%$ of injurious assaults committed by inpatients were against staff. Inpatient aggression is also a costly problem beyond staffing issues. An often-cited study estimated that assaults resulting in injury in a forensic inpatient facility in 1988 cost $\$ 5719$ per event in employee damages [4].

A small group of patients account for the majority of violent incidents, with less than $10 \%$ of patients responsible for $70 \%$ of assaults in one study of prevalence [5]. As a small number of individuals are responsible for a majority of violent incidents, and these incidents have substantial fiscal costs to the system, as well as detriments to the work environment and safety of employees, it is beneficial to determine the characteristics of those few individuals.
While research is contradictory relating specific clinical syndromes to inpatient violence [5-8], most studies exclude malingerers (those feigning or exaggerating a disorder) in their sample. Malingering has a negative impact on other aspects of the criminal and mental health systems, which are reviewed below, and should therefore be evaluated as a potential contributor to violence in inpatient facilities.

\section{Impact of malingering on the court system}

It is estimated that $19 \%$ to $54 \%$ of criminal defendants malinger their mental illness in evaluations, such as those to determine competency for trial [10-12]. To roughly calculate the costs of unnecessary treatment for those malingering the required mental illness, Stensland, Watson, and Grazier [13] concluded that the daily cost of inpatient psychiatric care within the United States averaged between $\$ 850$ and $\$ 1,100$ per person, varying by diagnosis. In the sample of malingerers in this study, the average length of stay was 110 days, suggesting that their unnecessary hospitalization had an 
average cost between $\$ 93,500$ and $\$ 121,000$ per person. Furthermore, determining the financial burden of malingerers requires including the cost of competency evaluations, as well as transportation from court to inpatient facilities [9].

Research on manipulative personality disorders indicates associations with increased inpatient aggression [7,14-16]. We hypothesize that the trait of manipulativeness, inherent in malingerers feigning symptoms to manipulate the legal system, would also be associated with increased aggression.

\section{Diagnoses and aggression}

Specific clinical syndromes are associated with more inpatient assaults. Particularly, there is a direct relationship between the presence of psychosis and perpetration of assaults while hospitalized [5,17-26]. However, other research has demonstrated reduced assault rates in patients diagnosed with a psychotic disorder [7]. Similarly, mood disorders have some support for a relationship to increased inpatient assaults [5,18,26,27]. Staggs [2] found that inpatient assaults against staff were five times more frequent on geriatric psychiatric units compared to adult psychiatric units, possibly due to paranoia and confusion related to dementia. Alternately, some studies suggest there are no significant differences in inpatient assaults by clinical syndrome [6,8], making this an important area to explore.

Unlike syndromes, specific personality disorders are related to inpatient aggression. The association between a personality disorder and inpatient assaults suggest that personality needs may motivate aggression regardless of impairment in reality testing [7,18,21,26,28-31]. The antisocial personality disorder (ASPD) diagnostic criteria specifically speaks of aggressive acts towards others and is related to inpatient assaults [15,28,30-32]. There is also evidence of increased inpatient assaults amongst other personality disorders with notably manipulative traits [14-16]. However, the interaction of personality disorders and serious mental illness in inpatient assaults is unclear. A meta-analysis of studies on patients with psychosis found that personality disorders typically went undiagnosed [23]. Because psychosis has the most support related to assaults, the lack of personality diagnosis is problematic in understanding the relationship between serious mental illness, personality disorders and assaults.
The act of malingering is defined by manipulation for secondary gain (such as dismissal of charges or financial gain) [34]. As manipulation is a trait also seen in some of the personality diagnoses linked to increased inpatient aggression, this suggests that a relationship between malingering and inpatient assaults may exist. Assaultive behavior could be a tool used to meet their perceived needs, similarly to malingering symptoms.

While there is a paucity of research on malingerers and inpatient assaults, one small study compared a sample of 18 patients at a high security hospital determined to be malingering insanity, to a sample of hospitalized non-malingerers [35]. There was a greater prevalence of ASPD in their sample of malingerers, and they were significantly more verbally and physically assaultive while hospitalized. Many had violent offenses prior to hospitalization and continued to present other management problems, such as drug dealing and sexual relations with staff. While this is a single study of a small sample with unclear identification of malingerers, it suggests further examination of the impact of malingering on inpatient behavior is warranted. The research reviewed indicates these traits are significantly related to assaults by inpatient psychiatric patients.

There is clearly a need to reduce the number of assaults in inpatient units. One way to do that is to reduce the number of aggressive patients in units, and particularly those who are utilizing resources unnecessarily. The current study hypothesizes that those who are malingering mental illness but are put in inpatient settings, contribute significantly to inpatient assaults. If we are able to better identify these individuals, we may be able to significantly decrease the levels of aggression in inpatient settings.

\section{Aim of the Study}

The present study aims at gathering information to determine the relationship of malingering to inpatient aggression so as to reduce the violence, particularly perpetrated by malingerers, in forensic settings.

\section{Methods}

\section{Participants}

Valid data was obtained from 4,918 participants admitted to a large state psychiatric hospital in Florida over the span of eight 
years, July 1, 1997 to June 30, 2005, before the data tracking system was terminated. All patients with the primary diagnosis of malingering were selected for use in the study $(2.3 \%)$.

The participants were identified as malingering $(n=112)$ and were matched with non-malingering patients $(n=112)$ with the same legal status, with equal lengths of hospitalization (or opportunities to assault), closest age upon admission, and gender (when possible). The average age of admission amongst the malingering patients was 33.82 years ( $M d n=32.5, \mathrm{SD}=9.9$, range: $17-68)$. The average age of admission amongst the non-malingering patients was 33.80 years ( $\mathrm{Mdn}=32, \mathrm{SD}=10.53$, range: 18 - 77). Using the date admitted to the hospital, groups were matched by their length of hospitalization. They both averaged a length of hospitalization of 100.63 days, with a maximum of 685 days (approximately 1.88 years) and a minimum of six days.

Of the malingering patients, $97.3 \%$ were males $(n=109)$ and $2.7 \%$ were females $(n=3)$. Of the non-malingering patients, $83.9 \%$ were male $(n=94)$, while $16.1 \%$ were female $(n=18)$. While there are significantly more women in the non-malingering group, the results indicate that women did not have significantly less participants who assaulted at least once, and women do not have significantly lower assault rates than men. It is suspected that racial classifications were underrepresented and misclassified. One indication of this was that the 50 earliest admissions in the full dataset were all classified as "black" or "white" suggesting that subgroupings were introduced later in the data entry at the hospital. In the group of malingerers $52.7 \%$ were identified as "Black" ( $n=59)$, $36.6 \%$ as "White" ( $\mathrm{n}=41), 2.7 \%$ as "Hispanic" $(\mathrm{n}=3)$, and $8 \%(\mathrm{n}=$ $9)$ did not have a racial classification recorded. Within the non-malingering group $61.6 \%$ were identified as "Black" ( $n=69$ ), $33.0 \%$ as "White" ( $n=37), 0.9 \%$ as "Hispanic" ( $n=1), 1.8 \%$ as "Asian" ( $n=2)$, and $2.7 \%(n=3)$ were missing data. See table 1 for a comparison of primary and secondary diagnoses, and table 2 for personality disorder diagnoses in the paired groups. It is notable that in the malingering group, the most common secondary diagnosis was a substance abuse disorder $(n=56)$.

\begin{tabular}{|c|c|c|c|c|}
\hline & \multicolumn{4}{|c|}{ Percentage of Diagnosis (n) Axis 1 } \\
\hline & \multicolumn{2}{|c|}{ Primary Diagnosis } & \multicolumn{2}{c|}{ Secondary Diagnosis } \\
\hline Diagnosis & Non-Malingerers & Malingerers & Non-Malingerers & Malingerers \\
\hline Psychotic Disorders & $47.3(53)$ & - & $.9(1)$ & $7.1(8)$ \\
\hline Mood Disorders & $9.8(11)$ & - & $1.8(2)$ & $3.6(4)$ \\
\hline Mixed Mood and Psychotic & $41.1(46)$ & - & $2.7(3)$ & $1.8(2)$ \\
\hline Not Mood or Psychotic & $1.8(2)$ & - & $52.7(59)$ & $59.8(67)$ \\
\hline None & - & - & $42.0(47)$ & $27.7(31)$ \\
\hline Malingering & - & $100(112)$ & - & - \\
\hline
\end{tabular}

Table 1: Primary and secondary diagnoses.

\begin{tabular}{|c|c|c|}
\hline & \multicolumn{2}{|c|}{ Axis 2 Personality Disorder } \\
\hline Diagnosis & Non-Malingerers & Malingerers \\
\hline Antisocial & $17.0(19)$ & $67.9(76)$ \\
\hline Borderline & $1.8(2)$ & $2.7(3)$ \\
\hline Dependent & $1.8(2)$ & - \\
\hline Histrionic & - & $.9(1)$ \\
\hline Schizoid & - & $.9(1)$ \\
\hline Not Otherwise Specified & $2.6(4)$ & $5.4(6)$ \\
\hline None & $75.9(85)$ & $22.3(25)$ \\
\hline
\end{tabular}

Match procedures

From the full sample of participants, 112 patients with the DSMIV-TR condition of malingering were selected [34]. First assault rates from the entire sample of patients $(\mathrm{N}=4918)$ was used to establish base rates of assaults for those identified as malingering and the rest of the population. In order to compare the aggression level of malingerers and non-malingering patients who have had the same opportunities to engage in aggression, each patient with a malingering diagnosis was then matched with a non-malingering patient.

Table 2: Personality disorder prevalence. 
Matches were sorted by length of stay (days of opportunity to assault), within that it was further narrowed to those with the same legal status (such as pretrial or civil commitment), and of those options the match selected was the one with the closest age upon admission, and same gender when possible. These selections were done in an effort to avoid other variables that have previously been determined to impact inpatient aggression, such as age and legal status. On average, the pairs have an age difference of less than one year $(\mathrm{M}=.02, \mathrm{SD}=7.01)$.

\section{Measures}

Primary diagnoses

Because these data were collected for eight years, the diagnostic criteria of the DSM IV-TR were used [34]. In this psychiatric facility, all diagnoses were made by the treating psychiatrist, informed by the full multidisciplinary team. The final diagnosis at discharge was coded for each participant. Evidence through psychological testing and comparisons of reported symptoms and behavioral observations, were used by the team to inform diagnosis.

Malingering was identified through a combination of symptom validity testing and behavior while under 24-hour observations, as designed by the psychologist on the team. Assessments were customized for each case and typically included use of the Miller Forensic Assessment of Symptoms Test, Structured Interview of Reported Symptoms, Test of Memory Malingering, and objectives measures of personality and intelligence such as the Minnesota Multiphasic Personality Inventory and Wechsler Adult Intelligence Scale. For example, one case was exaggerating cognitive and memory impairment while committed as incompetent to proceed to trial. He stated he was unable to restate his charge (Grand Theft Auto) after prompting by his psychologist. Testing indicated that the patient was not giving full effort, and his cognitive capacity was corroborated by observed behavior, including his success winning the Hearts card game tournament against staff on his unit. This evidence was presented to the team, which resulted in a diagnosis change.

Malingering cases primarily feigned psychosis and cognitive impairment. The primary diagnosis of malingering indicates that the psychiatrist found their primary presenting issue to be exaggerating symptoms. This is a specific volitional act that is identified as their primary obstacle in functioning, an act which is absent in the comparison group. It does not preclude the presence of other comorbid conditions, such as an anxiety disorder or a psychotic condition in remission. But as the primary diagnosis, this indicates that despite any other conditions, the primary obstacle to their functioning is the volitional choice to manipulate their presentation for secondary gain. The primary diagnosis identified by the psychiatrist was also used for the comparison group, which was also informed with testing and observations.

\section{Comorbid personality disorders}

The literature described above suggests personality disorders may contribute to assaults by psychiatric inpatients. Therefore, personality disorders were included, as diagnosed using specific criteria within the DSM-IV-TR. While there are a wide range of personality disorders, within this sample of malingering patients, $67 \%$ of personality disorders were ASPD. As such this sample is unlikely to be a good representation of other individual diagnoses in the analyses (such as Borderline and others). As research indicates, due to the degree of functional impairment caused by clinical syndromes, personality disorders are under-identified in genuine psychiatric patients, and this would be better able to be detected when symptoms of their primary diagnosis are in remission [33].

\section{Assaults}

This hospital records assaults that were an attempt to cause physical injury to another, regardless of the outcome. Nurses onduty were required to complete an incident report with observers of the incident. More serious physical attempts requiring staff intervention to establish safety would have a greater probability of being documented than attempts that represented little risk to safety. These events were also coded to note roles of victim or aggressor, if the assault targeted an employee or patient, and injury outcome of the event.

There were three outcome variables assessed, including whether the subject assaulted or not, number of assaults, and assault rate per day of hospitalization.

\section{Results}

Demographic variables were first explored to examine their relationship to our measures of assaults. Results of a logistic regres- 
sion indicated that whether a subject committed an assault or not was not significantly impacted by their gender, race, or age, $\mathrm{X}^{2}(5)=$ $3.1, \mathrm{p}>$.05. Next, we ran a Poisson regression using the natural log of the length of hospitalization as an offset variable to convert the number of assaults into a rate. Again, we examined whether gender, race or age were significant predictors. This model was significant (Omnibus test: $\left.\mathrm{X}^{2}(3)=14.01, \mathrm{p}=.003\right)$, but age was the only significant factor. Younger individuals had a higher assault frequency than older individuals $(\beta=-.03, \mathrm{SE}=.01, \mathrm{p}<.01)$. For every oneyear increase in age, the assault rate decreases by .97 (95\% CI: .95.99). For this reason, age was included in every analysis as a possible independent variable, while race and gender were excluded.

\section{Base rates of assault}

The sample of malingerers $(n=112)$ committed an assault once every 3 months on average ( $\mathrm{M}$ assaults per day $=.011, \mathrm{SD}=.023$ ). The remaining non-malingerers $(n=4806)$ committed an assault once every 5 months on average (M assaults per day $=.007, \mathrm{SD}=$ .017). However, these groups vary based on age, legal status, and diagnosis, which are known to be associated with inpatient assaults $[7,36]$. Therefore, matched samples were used to control for the additional variables and compare those identified as malingering to those presenting with a genuine mental illness.

\section{Those who assault}

There were significantly more malingerers who assaulted $(41 \%)$ than genuine psychiatric patients $\left(27 \% ; \chi^{2}(1)=5.10, p=\right.$ .02 , odds ratio $=1.91,95 \% \mathrm{CI}: 1.09,3.34$ ). Thus, malingerers are 1.9 times more likely to commit at least one assault than genuine psychiatric patients.

\section{Number of assaults}

The distribution for the number of assaults was not normal, therefore we used non-parametric statistics. The average number of assaults committed by malingering patients $(\mathrm{M}=.64$, $\mathrm{SD}=.97)$ was not significantly different than the number of assaults committed by the genuinely mentally ill $(\mathrm{M}=.58$, $\mathrm{SD}=1.47$; Wilcoxon Signed Rank Test, $\mathrm{Z}=-1.5, \mathrm{p}=.13, \mathrm{r}=.14$ ). As our data were not normally distributed, we assessed outliers via the median absolute deviation, which is a better method for assessing outliers than standard deviation for skewed data [37]. Four of the matched-pairs included a number of assaults that were greater than three absolute deviations above the median, indicating they were statistical outliers. When these outliers were removed, malingering patients $(\mathrm{M}=.58, \mathrm{SD}=.86)$ committed significantly more assaults than mentally ill patients $(\mathrm{M}=.38, \mathrm{SD}=.81$; Wilcoxon Signed Rank Test, $\mathrm{n}$ $=108, \mathrm{Z}=2.24, \mathrm{p}<.01, \mathrm{r}=.22$ ). However, because all participants within each diagnostic group did not have equal opportunity to stay to accrue assaults, no additional analyses were conducted on the raw count data. The sample of malingerers after the removal of outliers committed 63 assaults $(n=108)$. The match group committed 41 assaults $(n=108)$.

\section{Assault rate intensity}

Previous analyses examined whether assaults happened or not and how many assaults happened, but they did not adequately capture patients that stayed for a short amount of time with a high intensity of violence. For this we examined the assault rate per day of hospitalization. Malingerers $(\mathrm{M}=.011, \mathrm{SD}=.02)$ assault significantly more frequently per day hospitalized (Wilcoxon Signed Rank Test, $\mathrm{Z}=-2.34, \mathrm{p}=.02, \mathrm{r}=.22$ ) than genuinely mentally ill patients $(\mathrm{M}=.005, \mathrm{SD}=.01$; See figure 1$)$.

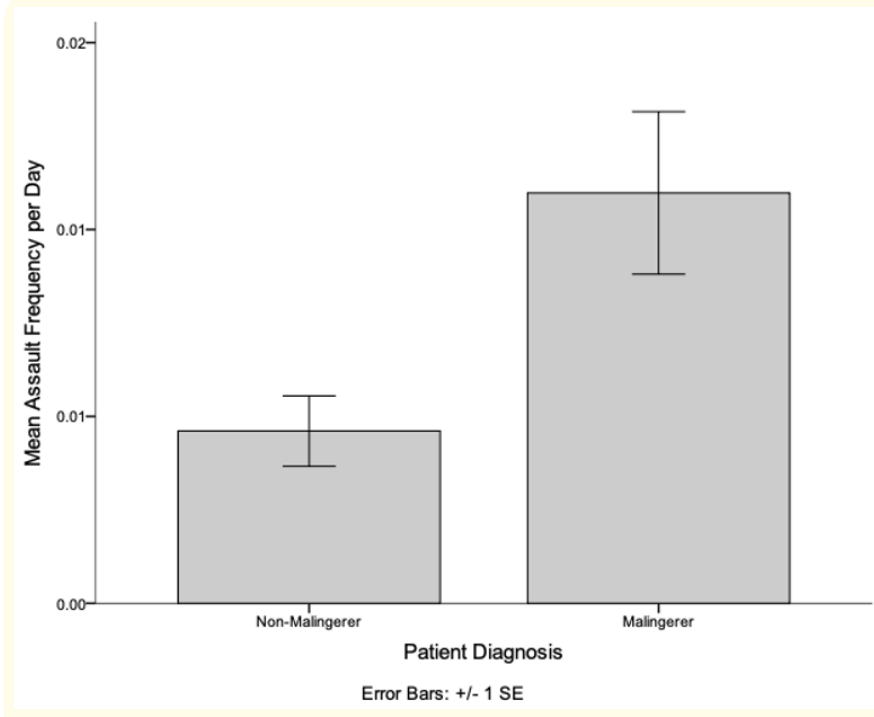

Figure 1: Assault frequency per day in the matched groups. 
To model which malingering individuals were more likely to have high assault rates, we used a Poisson regression model corrected for overdispersion [38] with the predictors of age and personality disorder type for malingerers. We used the natural log of length of hospitalization as an offset in order to transform the raw count data into a rate. The model was significant, $X^{2}(4)=11.41, \mathrm{p}=$ 0.02 . Age was again a significant predictor of assaults $\left(X^{2}(1)=3.84\right.$, $\mathrm{p}=.05$ ) with younger patients assaulting more frequently during their stay $(\beta=-.03, \mathrm{SE}=.01, \mathrm{p}<.01)$. For every one-year increase in age, the assault rate decreases by .97 (95\% CI: .94 - .99) in malingerers, see figure 2 . Comorbid personality disorder type did not impact the assault rate in malingerers $\left(X^{2}(3)=3.42, \mathrm{p}=.33\right)$.

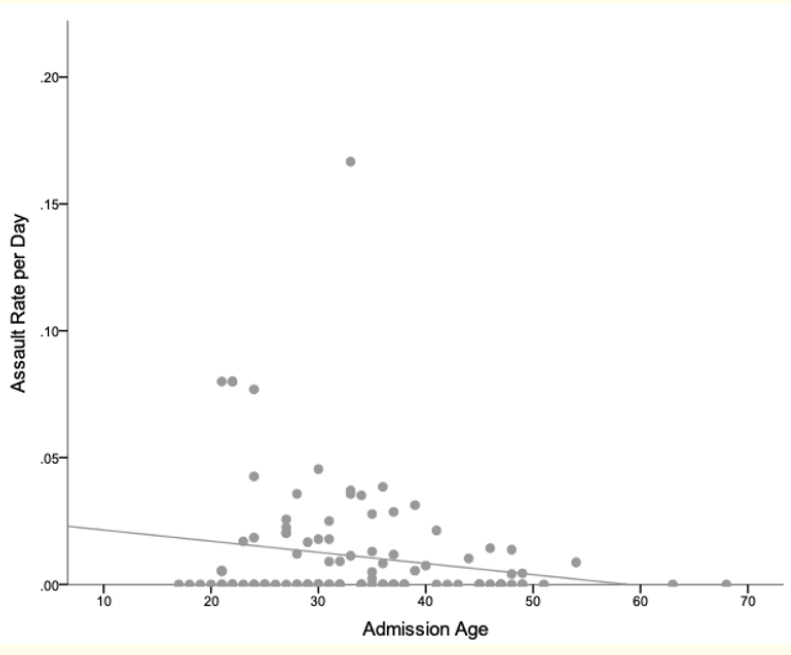

Figure 2: Relationship between age and assault rate for malingerers.

Perhaps the reason personality disorder was not a significant predictor in the malingering group was the high number of malingerers with a comorbid personality disorder (77.7\%) and high percent of malingerers committing assaults (41\%). The diagnoses of malingering and personality disorder are highly correlated $(r$ $=.55, \mathrm{p}<.01$ ), suggesting they may explain the same variance in assault rate. Young malingerers are predicted to commit a higher frequency of assaults.

Among the mentally ill patients, there were four individuals whose standardized residuals indicated they were statistical outli- ers $($ residual $>|2|)$. These were excluded from the model. In the Poisson regression for mentally ill participants, age, personality disorder, and clinical syndrome were entered as predictors and this model was significant $\left(X^{2}(7)=29.32, \mathrm{p}<.01\right)$. For mentally ill patients, age $\left(X^{2}(1)=8.61, \mathrm{p}<.01\right)$ and personality disorder type $\left(X^{2}(2)=10.80, \mathrm{p}<.01\right)$ were significant predictors, but clinical syndrome was not $\left(X^{2}(3)=2.57, \mathrm{p}=.46\right)$. For each year increase in age in mentally ill patients the assault rate decreases by $.95(95 \% \mathrm{CI}$ : $.92-.98 ; \beta=-.05, \mathrm{SE}=.02$ ).

Additionally, mentally ill patients without a comorbid personality disorder were less likely to assault $(\beta=-1.246, \mathrm{SE}=.3912, \mathrm{p}=$ .001 , adjusted $\beta=.288,95 \%$ CI: $1.34, .619$ ). No other categories of personality disorder were significantly predictive of assault rate. This is likely due the small sample sizes of personality disorders (Personality Disorder NOS ( $n=6)$, BPD ( $n=2)$, ASPD $(n=16)$ ). However, the mean assault rate in those with ASPD (M = .0115, SE $=.005$ ) appears descriptively higher than those with Personality Disorder NOS (M = .008, SE = .003), and those without a personality disorder $(\mathrm{n}=83, \mathrm{M}=.003, \mathrm{SE}=.001)$. Therefore, this should be further explored in a sample with a better representation of personality disorders. In the mentally ill group, older patients without a comorbid personality disorder are the least likely to commit a higher frequency of assaults.

\section{Comorbid personality disorders}

Malingerers $(77.7 \%)$ are significantly more likely to be diagnosed with a comorbid personality disorder than the genuinely mentally ill $(24.1 \%), \chi^{2}(1)=64.306, p<.001$, odds ratio $=10.956$, CI $[5.889,20.38]$. This indicates that malingerers are nearly 11 times more likely to receive a diagnosis of having a comorbid personality disorder than the genuinely mentally ill. This is consistent with research that personality disorders are chronically underidentified in those with significant clinical symptoms such as psychosis $[33,39]$. One study found that with testing, the prevalence rate of personality pathology was $81 \%$ in a sample of inpatients with schizophrenia, but prevalence is as low as $4.5 \%$ diagnosed in the population $[33,40]$. Research supports that personality disorders are unlikely to be diagnosed in patients with serious mental illness. Therefore, we suspect personality disorders are more prevalent than is diagnosed in our sample, and personality disorders 
alone are not the underlying cause of the increased violence we see in malingerers. However, we attempt to control for this possibility in the analysis below.

To determine whether a comorbid diagnosis of a personality disorder influences whether or not a person assaults rather than a diagnosis of malingering, we ran an analysis using only people with a comorbid personality disorder. This analysis included 27 genuinely mentally ill patients ( $\mathrm{M}$ age $=33.33, \mathrm{SD}=9.422,88.9 \%$ male, $\mathrm{M}$ length of hospitalization $=115.44, \mathrm{SD}=108.09$ ) and 87 malingering patients $(\mathrm{M}$ age $=32.69, \mathrm{SD}=8.335,98.9 \%$ male, $\mathrm{M}$ length of hospitalization $=95.87, \mathrm{SD}=116.149$ ). Among participants with a comorbid personality disorder, there was no difference in whether or not someone committed an assault between malingerers $(44.8 \%, \mathrm{n}=39)$ and the genuinely mentally ill $\left(48.1 \%, \mathrm{n}=13 ; \chi^{2}(1)\right.$ $=.092, \mathrm{p}=.762$ ). Although the mean assault rate for malingerers was higher ( $M=.0128, \mathrm{SD}=.02528)$, it was not significantly different than the assault rate in the sample of mentally ill with a personality disorder $(\mathrm{M}=.0105, \mathrm{SD}=.01437$; Mann-Whitney $\mathrm{U}=-.28, \mathrm{p}$ $=.779$ ). However, these results should be interpreted with caution due to the large difference in sample sizes between the groups. As group sizes become more unequal, the power to detect an effect decreases. Thus, these tests may not have enough power to detect an effect if there truly is a group difference. While we cannot rule out personality disorders impacting assault rates, it is notable that the manipulative traits leading to a diagnosis of malingering increases the probability of being diagnosed with personality pathology, and those with personality disorders may be more inclined to malinger when facing criminal charges.

\section{Discussion and Conclusion}

This study examined the role of malingerers in inpatient assaults. The findings of this study demonstrate that more malingerers committed assaults (41.1\%) during the matched days than psychiatric patients who were not malingering (26.8\%). These findings reflect a simplistic paired comparison, where many patients engage in aggression at least once as they adjust to an institutional setting and to avoid victimization by others. Research indicates that a significant number of assaults occur within the first two, five, and thirty days following admission $[5,41,42]$. It is possible that assaults early in a hospitalization serve the purpose to establish themselves in the social group and avoid victimization by more dominant individuals. Alternately, change in assaults over time may reflect a response to psychiatric treatment. Assuming that most patients in treatment will demonstrate improvement of symptoms related to violence over time, it is likely that those feigning mental illness will not demonstrate similar gains and further unwarranted hospitalization would only continue their violent behavior.

As predicted, malingerers committed more than just the single assault commonly associated with early admission to institutional setting; they committed a higher raw number of assaults than the genuine psychiatric patients and assaulted at a higher rate per day during their hospitalizations. Additional factors that exacerbated assault rates were age and comorbid personality disorders. In the malingering sample, younger patients demonstrated a higher frequency of assaults throughout their stay. While comorbid personality disorder was not a significant predictor of assault rate in malingerers, it was found that there was a significant correlation between being diagnosed as malingering and having a personality disorder, suggesting they both may play a role in describing the variance in assault rate. Similarly, in the sample of mentally ill participants, age and comorbid personality disorder are significant predictors, with a higher assault rate from those younger and diagnosed with a comorbid personality disorder. We suspect that this effect is being driven by those with ASPD, but our sample of mentally ill diagnosed with ASPD was too small to definitively make this claim. This is something that warrants further research. Malingerers who assaulted had a large number diagnosed with ASPD (71.7\%). Examining those with high assault frequency suggests youth, ASPD, and malingering are indicators of assault risk.

It is notable that malingering shares many qualities with ASPD. One criteria for ASPD is failure to conform to social norms with respect to lawful behavior; malingerers are likely motivated to engage in malingering to avoid the consequences of unlawful behavior. Another criteria of ASPD is deceitfulness and conning others for personal profit. By making the choice to feign or exaggerate mental illness for secondary gain, malingerers are also conning others for profit. The phenomenon of manipulativeness is present in both disorders and may explain assaults at the hospital as another method enlisted for personal gain. 
Inpatient assaults were associated with ASPD. Prior research supports that among those hospitalized while awaiting trial, inpatient assaults were generally volitional and motivated by personality disorder and legal circumstance [7]. In those with mental illness, the presence of positive symptoms of mental illness combined with traits of psychopathy mediated the relationship between anger and aggression, increasing the likelihood of inpatient assaults [43]. This is very similar to the motivation described above for antisocial malingerers. Inpatient assaults can serve the purpose of maintaining dominance, exploiting others, attempting escape, or merely continuing to receive pleasure from engaging in violent behavior.

However, research suggests that personality disorders are under-identified in psychiatric patients $[33,39,40]$. Therefore, it is likely that our sample of genuinely mentally ill patients has a higher rate of personality disorders than our data suggests. In other words, we suspect personality disorder alone is not the underlying cause of the increased violence we see in malingerers. More research is needed to explore the prevalence of comorbid personality disorders in inpatient facilities. However, we interpret these results to support the role of malingering in inpatient assaults. It is possible that ASPD and malingering are explaining the same variance in assaults, but personality disorders are more easily identified than malingering. The DSM-5 has set criteria for ASPD, so as long as behavioral observations and evidence of a juvenile conduct disorder are available, it is possible to diagnose [48]. However, malingering is listed as a V-code with less delineated criteria. Psychological assessments are available, aimed at detecting specific attempts at malingering, such as feigning rare combinations of symptoms. However, they require comparisons of behavioral observations with collateral data, and evidence of a secondary gain, to identify malingering. It is possible these factors make it easier to diagnose someone with ASPD than malingering.

In both samples youth predicted higher assault rates. Research supports reduced antisocial behavior in men as age increases, and also reduced rates of qualifying for the diagnosis of ASPD as age increases [36,45-47]. It is therefore not surprising that youth in malingerers would also be associated with increased inpatient assaults.
This research furthered the findings of the only other study on malingering and inpatient assaults. That study found that malingerers of insanity $(n=18)$ had a greater prevalence of ASPD diagnosis and inpatient assaults [35]. This study, of a much larger sample, was primarily of those malingering incompetence to stand trial (98.5\%) and found they were more assaultive than the genuinely mentally ill. Similarly, we found that assaults were more frequent in younger malingering patients with ASPD.

\section{Implications}

The differences in raw count of assaults can be directly translated to costs per incident during the unnecessary hospitalization of malingerers. As mentioned previously, the prolific and aggressive behavior displayed by malingerers has financial implications and the psychological impact of burnout and trauma on genuine patients. This further supports the conclusion that malingerers not only create superfluous financial strain on institutions but create dangerous environmental conditions that are consistently evidenced throughout their hospitalization.

Changes should be considered in the way we identify and manage malingerers in inpatient settings. The present study identified 112 patients whose primary condition was malingering, from 4,918 patients treated over the course of eight years. Not including individuals with secondary or tertiary identification of malingering, the calculated costs of treating this group of patients for their average length of stay (110 days) would equate to minimum predicted cost of $\$ 10,472,000$ [13] plus $\$ 411,768$ in assault related costs (for 72 assaults by 112 malingerers; [4]). This total is extortionate and irresponsible as practitioners.

APA ethical guidelines require that forensic psychologists use appropriate methods of examination and examine collateral sources in these evaluations [48] (Guideline 8.03 and 9.01). Why then are so many malingerers being committed as if truly mentally ill, and admitted for costly inpatient treatment? What are the circumstances surrounding appointment of experts in these cases that could contribute to the problem? The Supreme Court of Florida enacted an administrative order in 2017 , to advance the selection process 
in finding expert witnesses, while reducing the cost of evaluations [49]. Professionals have left the Board of Examiners in their circuits, resulting in less available experts. This order noted maximum payment rates, reevaluation rates, and suggested only one expert be used unless both sides do not agree upon the findings. In personal communication with these authors, forensic evaluators in the state have noted a number of problems with the implementation of this order. Some circuits pay psychiatrists more than psychologists, and do not reimburse additionally for malingering testing. Yet, psychologists conduct psychological testing, including assessments of malingering, in competency evaluations significantly more frequently than psychiatrists [50,51]. Some circuits reportedly do not allow an expert to charge for their time while testifying or waiting to testify, causing some to be subpoenaed for phone testimony and left waiting for hours on the phone. These practices discourage seasoned forensic professionals from participating in routine competency evaluations, which may identify malingerers. When circuits fail to encourage evaluators to detect malingering, the state pays far more money long term in expensive unnecessary hospitalization, rather than if they invested in a better-quality competency evaluation up front. Hopefully awareness of the long-term consequences of these decisions will result in adjustments to policy. These concerns are likely present in other states conducting evaluations of defendants.

While it would also be recommended to initiate malingering screenings within the first few weeks of admission, it is notable that even short unnecessary hospitalizations are problematic. In addition to transportation and security costs, a significant number of assaults occur within the immediate days following admission, typically between three and 30 days $[5,41,42]$. When an admitted patient is found to be malingering, hospitals are then dependent on the County for transportation back to the respective correctional setting. These arrangements can take days, and even months, depending on the resources associated with that county. As such, it is recommended that further resources are dedicated to independent community evaluations [52,53]. By allocating more resources to evaluations prior to admission they can prevent inappropriate, hazardous, and exorbitantly expensive hospital conditions.

\section{Limitations and Future Directions}

Although this study fills an important gap in understanding inpatient assaults, it is not without limitations. While both groups were matched on length of stay, they did not enter the hospital on the same day or to the same units, so environmental influences may have differed between groups. Final determinations on diagnoses were made by psychiatrists identifying the primary presenting issue. Some patients may have been malingering mental illness as it related to legal issues, such as feigning memory impairment for the crime, but may have presented symptoms without exaggeration in other context resulting in a primary diagnosis that was not malingering. In addition, personality disorders may be more prevalent than the data suggested, due to symptoms like psychosis impairing the expression of the personality being detected by practitioners. Diagnosis strength may also have been impacted by varying amounts of records available to staff from the community and prior hospitalizations, determined by how much contact each patient has had with the mental health system. We cannot rule out that personality disorders may be an underlying diagnosis that leads to a high assault rate, although this should be explored with a larger sample of genuinely mentally ill patients that also have a comorbid personality disorder.

Future studies could examine the assault rates of malingerers depending on the sentence they are facing if found competent to proceed to trial. Would they make a greater effort to appear mentally ill and "out of control" of their behavior if facing a life sentence? Changes in assaults depending on how long the malingerer has been able to remain undetected in the hospital would be interesting to examine. It would also be interesting to examine the role of treatment and treatment compliance on assaults by those who are genuinely mentally ill.

\section{Bibliography}

1. Rippon T J. "Aggression and violence in health care professions". Journal of Advanced Nursing 31.2 (2000): 452-460.

2. Staggs V S. "Trends, victims, and injuries in injurious patient assaults on adult, geriatric, and child/adolescent psychiatric units in US hospitals, 2007-2013". Research in Nursing and Health 38.2 (2015): 115-120.

3. Owen C., et al. "Violence and aggression in psychiatric units". Psychiatric Services 49.11 (1998): 1452-1457. 
4. Hunter M and Carmel H. "The cost of staff injuries from inpatient violence". Psychiatric Services 43.6 (1992): 586-588.

5. Barlow K., et al. "Prevalence and precipitants of aggression in psychiatric inpatient units". Australian and New Zealand Journal of Psychiatry 34.6 (2000): 967-974.

6. Aiken G. "Assaults on staff in a locked ward: Prediction and consequences". Medicine, Science and the Law 24.3 (1984): 199-207.

7. Costopoulos JS. "Aggression in an Inpatient Psychiatric Facility: A retrospective longitudinal study". Journal of Forensic Psychology Research and Practice 19.3 (2019): 242-259.

8. Oulis P., et al. "Aggression among psychiatric inpatients in Greece”. Psychopathology 29.3 (1996): 174-180.

9. Miller RD. "Hospitalization of criminal defendants for evaluation of competence to stand trial for restoration of competence: Clinical and legal issues". Behavioral Sciences and the Law 21.3 (2013): 369-391.

10. Ardolf BR., et al. "Base rates of negative response bias and malingered neurocognitive dysfunction among criminal defendants referred for neuropsychological evaluation". The Clinical Neuropsychologist 21.6 (2007): 899-916.

11. Lewis J L., et al. "Screening for feigned psychiatric symptoms in a forensic sample by using the MMPI-2 and the structured inventory of malingered symptomatology". Psychological Assessment 14.2 (2002): 170.

12. Mittenberg W., et al. "Base rates of malingering and symptom exaggeration". Journal of Clinical and Experimental Neuropsychology 24.8 (2002): 1094-1102.

13. Stensland M., et al. "An examination of costs, charges, and payments for inpatient psychiatric treatment in community hospitals". Psychiatric Services 63.7 (2012): 666-671.

14. Dougherty D M., et al. "Laboratory measures of aggression and impulsivity in women with borderline personality disorder". Psychiatry Research 85.3 (1999): 315-326.
15. Langton CM., et al. "Personality traits as predictors of inpatient aggression in a high-security forensic psychiatric setting: Prospective evaluation of the PCL-R and IPDE dimension ratings". International Journal of Offender Therapy and Comparative Criminology 55.3 (2011): 392-415.

16. Soliman A E D and Reza H. "Risk factors and correlates of violence among acutely ill adult psychiatric inpatients". Psychiatric Services 52.1 (2001): 75-80.

17. Amore M., et al. "Predictors of violent behavior among acute psychiatric patients: Clinical study". Psychiatry and Clinical Neurosciences 62 (2008): 247-255.

18. Biancosino B., et al. "Violent behavior in acute psychiatric inpatient facilities: A national survey in Italy". Journal of Nervous and Mental Disease 197 (2009): 772-782.

19. Cornaggia CM., et al. "Aggression in psychiatry wards: A systematic review". Psychiatry Research 189.1 (2011): 10-20.

20. Dack C., et al. "A review and meta analysis of the patient factors associated with psychiatric in-patient aggression". Acta Psychiatrica Scandinavica 127.4 (2013): 1-14.

21. Ketelsen R., et al. "Characteristics of aggression in a German Psychiatric hospital and predictors of patients at risk". Journal of Psychiatric and Mental Health Nursing 14 (2007): 92-99.

22. Muthukumaraswamy A., et al. "Aggression patterns and clinical predictors of inpatient aggression in a mental health low secure unit population". Journal of Psychiatric Intensive Care 4 (2008): 9-16.

23. Newton VM., et al. "Clinical decision-making about inpatient violence risk at admission to a public-sector acute psychiatric hospital". Journal of the American Academy of Psychiatry and the Law Online 40.2 (2012): 206-214.

24. Nolan K., et al. "Characteristics of assaultive behavior among psychiatric inpatients”. Psychiatric Services 54.7 (2003): 10121016. 
25. Omerov M., et al. "Incidents of violence in psychiatric inpatient care". Nordic Journal of Psychiatry 56 (2002): 207-213.

26. Raja M and Azzoni A. "Hostility and violence of acute psychiatric inpatients". Clinical Practice and Epidemiology in Mental Health 1 (2005): 11-19.

27. Knutzen M., et al. "Characteristics of psychiatric inpatients who experienced restraint and those who did not: A case-control study". Psychiatric Services 62.5 (2011): 492-497.

28. Bo S., et al. "The role of co- morbid personality pathology in predicting self-reported aggression in patients with schizophrenia". Comprehensive Psychiatry 54.5 (2013): 423-431.

29. Flannery R B., et al. "Characteristics of assaultive psychiatric patients: 20-year analysis of the Assaultive Staff Action Program (ASAP)". Psychiatric Quarterly 82.1 (2011): 1-10.

30. Lobbestael J., et al. "The relationship between adult reactive and proactive aggression, hostile interpretation bias, and antisocial personality disorder". Journal of Personality Disorders 27.1 (2013): 53-66.

31. Lobbestael J., et al. "The relationship between personality disorder traits and reactive versus proactive motivation for aggression". Psychiatry Research 229.1 (2015): 155-160.

32. Soliman A E D and Reza H. "Risk factors and correlates of violence among acutely ill adult psychiatric inpatients". Psychiatric Services 52.1 (2001): 75-80.

33. Newton-Howes G., et al. "The prevalence of personality disorder in schizophrenia and psychotic disorders: systematic review of rates and explanatory modelling". Psychological Medicine 38.8 (2008): 1075-1082.

34. American Psychiatric Association. "Diagnostic and statistical manual of mental disorders" (4th ed., text rev.). Washington, DC: American Psychiatric Publishing (2000).

35. Gacono CB., et al. "A clinical investigation of malingering and psychopathy in hospitalized insanity acquittees". Journal of the American Academy of Psychiatry and the Law Online 23.3 (1995): 387-397.
36. Holzer KJ and Vaughn MG. "Antisocial personality disorder in older adults: A critical review". Journal of Geriatric Psychiatry and Neurology 30.6 (2017): 291-302.

37. Leys C., et al. "Detecting outliers: Do not use standard deviation around the mean, use absolute deviation around the median”. Journal of Experimental Social Psychology 49.4 (2013): 764-766.

38. McCullagh P and Nelder J. "Generalized linear models". (2nd ed.). London: Chapman and Hall (1989).

39. Krueger R F and Tackett J L. "Personality and psychopathology: Working toward the bigger picture". Journal of Personality Disorders 17 (2003): 109-128.

40. Wei Y., et al. "Co-morbidity of personality disorder in schizophrenia among psychiatric outpatients in china: Data from epidemiologic survey in a clinical population". BMC Psychiatry 16.1 (2016): 1-8.

41. Gault C. "Incidents of battery in a forensic psychiatric setting: Development of statistical models for risk assessment". Auburn University, Alabama (1998).

42. Williamson R., et al. "Patient factors associated with incidents of aggression in a general inpatient setting". Journal of Clinical Nursing 23.7-8 (2014): 1144-1152.

43. Vitacco M J., et al. "Predicting short-term institutional aggression in forensic patients: A multi-trait method for understanding subtypes of aggression". Law and Human Behavior 33 (2009): 308-319.

44. American Psychiatric Association. "Diagnostic and statistical manual of mental disorders". (5th ed.). Arlington, VA: American Psychiatric Publishing (2013).

45. Lahey B., et al. "Annotation: The development of antisocial behavior: An integrative causal model". The Journal of Child Psychology and Psychiatry and Allied Disciplines 40.5 (1999): 669-682. 
46. Sampson RJ and Laub JH. "Life-course desisters? Trajectories of crime among delinquent boys followed to age 70". Criminology 41.3 (2003): 555-592.

47. Weissman MM. "The epidemiology of personality disorders: A 1990 update". Journal of Personality Disorders 1 (1993): 44-62.

48. American Psychological Association. "Specialty guidelines for forensic psychology". American Psychologist 68.1 (2013): 7-19.

49. Supreme Court of Florida. "Court Appointed Expert Witness Services In Florida's Trial Courts (FAC AOSC17-12)” (2017).

50. Borum R and Grisso T. "Psychological test use in criminal forensic evaluations". Professional Psychology: Research and Practice 26.5 (1995): 465-473.

51. Wright C., et al. "Assessment practices of professional psychologists: Results of a national survey". Professional Psychology: Research and Practice 48.2 (2017): 73-78.

52. Gowensmith W N., et al. "Lookin' for beds in all the wrong places: Outpatient competency restoration as a promising approach to modern challenges". Psychology, Public Policy, and Law 22.3 (2016): 293-305.

53. Steadman H J and Callahan L. "Reducing the Pennsylvania incompetency to stand trial restoration waitlist: More than just beds" (2017).

\section{Volume 4 Issue 6 June 2021}

(C) All rights are reserved by Julie $S$ Costopoulos., et al. 